\title{
La mise en place des cohortes en France : pourquoi, pour qui, comment et avec quels moyens?
}

\author{
Jean-Marie Goehrs ${ }^{1}$, Thomas Borel ${ }^{2}$, Dominique Costagliola ${ }^{3}$ et les participants à la table ronde $N^{\circ} 6$ \\ de Giens XXVI*
}

1 Université Pierre et Marie Curie, Paris, France

2 Sanofi, France

3 Inserm U943, Paris, France ; Université Pierre et Marie Curie, Paris, France

Texte reçu le 20 mars 2012 ; accepté le 4 juin 2012

Keywords:

cohort;

epidemiology;

public health

\begin{abstract}
Mots clés :
cohorte ;

épidémiologie ;

santé publique

Résumé - Une cohorte est la réunion d'un groupe de sujets partageant ensemble un certain nombre de caractéristiques, suivis longitudinalement, à l'échelle individuelle. Outils essentiels de l'épidémiologie, ces études permettent notamment d'argumenter sur l'éventuelle causalité entre une exposition et la survenue d'événements et d'orienter ainsi des politiques de santé publique.

En France, de nombreux projets de cohortes ont vu le jour ces dernières années. Parfois initiés par des équipes de recherche de façon autonome (ex. : « Étude épidémiologique auprès des femmes de l'Education nationale » ou « E3N »), ce sont de plus en plus souvent les autorités de santé qui en demandent la mise en place dans le cadre d'un plan de santé publique (ex. : la cohorte deterMinants and Evolution of AlzheiMer's disEase aNd relaTed disOrders ou Memento) ou pour répondre à un enjeu de sécurité sanitaire (ex. : la cohorte de patients porteurs d'un déficit héréditaire en protéines coagulantes ou France Coag) ou de suivi des médicaments après leur mise sur le marché (études « post inscription »).

La diversité des objectifs, des montages et des modes de gouvernance existants pour la réalisation d'une cohorte ont conduit les participants de la table ronde à s'interroger sur les enjeux que représente cet outil d'épidémiologie pour chacun des acteurs : les chercheurs, les régulateurs et les industriels. Un certain nombre de constats ont pu être partagés permettant de souligner les difficultés relatives à leur mise en place. Afin de rendre plus efficient la mise en place des cohortes, la table ronde de Giens a émis des propositions.
\end{abstract}

Abréviations : voir en fin d'article.

\footnotetext{
* Pour la liste des participants, voir en fin d'article.
} 


\section{Introduction}

Les études de cohorte consistent à observer la survenue d'évènements de santé dans le temps au sein d'une population définie. Elles permettent, notamment, d'évaluer les liens entre des facteurs dits d'exposition (démographiques, biologiques, comportementaux, environnementaux, génétiques...) d'une part et la survenue d'évènements de santé (maladie, marqueur biologique...) d'autre part. Elles sont devenues un outil irremplaçable pour étudier les effets sur la santé d'expositions peu fréquentes ou impossibles à évaluer correctement de manière rétrospective. Dans le domaine de la pharmaco-épidémiologie, elles sont aussi largement utilisées dans le but d'évaluer l'impact d'un médicament sur l'état de santé d'une population et du système de soins. C'est ainsi qu'en France, de nombreux projets de cohortes ont vu le jour ces dernières années.

Cependant, la méconnaissance des systèmes d'information ou des cohortes existantes, l'absence de règles de gouvernance préétablies, le manque de communication entre les acteurs et particulièrement les acteurs privés et publics, limitent sensiblement l'efficience de la réalisation de ces études épidémiologiques au regard des moyens nécessaires à leur réalisation. Dans un contexte où les compétences en épidémiologie sont rares, il est indispensable de s'interroger sur l'optimisation des ressources affectées à ce type de recherche.

Conscients de ces difficultés et après en avoir fait le bilan, les participants de la table ronde ont fait des propositions dans le but d'optimiser l'utilisation de cet outil épidémiologique.

\section{Les cohortes : un outil irremplaçable en santé}

La cohorte est un moyen d'investigation qui fait partie de la panoplie de base de l'épidémiologie. Les cohortes sont largement utilisées depuis fort longtemps dans des domaines très divers, et permettent de répondre à des questions de santé qui intéressent les chercheurs, les industriels et les autorités de santé. Les avantages principaux des cohortes par rapport à d'autres méthodes d'enquêtes sont qu'elles permettent : ${ }^{[1]}$

- la limitation des biais d'information, puisque les renseignements sur l'exposition sont enregistrés prospectivement et chez tous les sujets à risque d'exposition ;

- la prise en compte des pathologies concurrentes pour un même facteur de risque ou facteurs de risque concurrents pour une pathologie ;

- la possibilité de constituer des groupes de sujets exposés de taille suffisante pour étudier des expositions rares dans des cohortes de type exposés/non exposés ;

- la possibilité de tenir compte de phénomènes liés au temps (effet générationnel, effet de périodes...).

Différentes formes de cohortes existent permettant de répondre à des questions de santé publique plus ou moins ciblées.
Les cohortes centrées sur une maladie sont les plus courantes (effet d'une exposition ou d'une catégorie d'exposition sur une maladie et/ou un groupe de population souffrant de celle ci). Parmi celles à visée de recherche sur une catégorie de facteurs de risque, on peut citer à titre d'exemples :

1- La cohorte nationale deterMinants and Evolution of AlzheiMer's disEase aNd relaTed disOrders (MEMENTO) ${ }^{[2]}$ a été récemment officiellement lancée, mis en œuvre par le Groupe méthodologies Alzheimer (GMA) dans le cadre du plan Alzheimer 2008-2012. Elle suivra pendant 5 ans 2300 patients présentant des signes cliniques - notamment des troubles de mémoire - pouvant évoquer une forme débutante de la maladie d'Alzheimer ou une maladie apparentée. MEMENTO vise deux objectifs principaux. Le premier consiste à mieux comprendre la maladie afin d'accélérer le développement de nouveaux traitements. Le second est de servir de plateforme nationale pour le développement de projets de recherche.

2- Une autre cohorte spécialisée, qui s'apparente plus à un registre, est le projet « Réseau FranceCoag », ${ }^{[3]}$ qui suit les malades atteints de maladies hémorragiques héréditaires. En effet, suite au drame du sang contaminé et à l'instar de certains pays européens ou d'Amérique du Nord, un programme national de surveillance des patients hémophiles traités par des produits dérivés du sang (SNH) a vu le jour en France en 1994. Cette surveillance s'est élargie depuis 2003 aux autres maladies hémorragiques héréditaires sévères dans le cadre d'un nouveau projet appelé Réseau FranceCoag. D'abord confiée à l'Institut national de la santé et de la recherche médicale (Inserm) [U720], la coordination de ce dispositif est assurée depuis janvier 2004 par l'Institut de veille sanitaire (InVS). Ayant toujours comme objectif la surveillance sanitaire de ces populations vis-à-vis des affections transmissibles, le Réseau vise également la connaissance exhaustive des caractéristiques et de l'évolution de ces populations.

On trouve également des cohortes qui ont pour but de répondre à plusieurs problématiques de santé à partir d'une population générale. C'est le cas par exemple de l'Étude épidémiologique auprès des femmes de l'Éducation nationale $(\mathrm{E} 3 \mathrm{~N}),{ }^{[4]}$ qui s'intéresse à l'étude des relations entre nutrition, hormones et survenue de cancers. Il s'agit d'une cohorte de 100000 femmes volontaires françaises nées entre 1925 et 1950 et suivies depuis 1990 adhérant à la Mutuelle générale de l'éducation nationale (MGEN), la plus grande cohorte française à ce jour. Les informations concernant d'une part leur mode de vie (alimentation, prise de traitements hormonaux...) et d'autre part l'évolution de leur état de santé, sont recueillies par auto-questionnaires tous les 2 ans depuis 1990. Elles sont complétées par des données biologiques, obtenues sur 25000 volontaires, à partir d'un prélèvement sanguin stocké à des fins de dosages ultérieurs (études cas-témoins dans la cohorte). E3N est la partie française de l'European prospective investigation into cancer (EPIC), vaste étude européenne coordonnée par le Centre International de Recherches sur le Cancer portant sur 500000 européens dans 
10 pays. Deux localisations cancéreuses sont étudiées en priorité du fait de leurs fortes incidences : le sein et le côlon-rectum.

D'autres cohortes réalisées en population générale s'intéressent à l'état de santé d'une population en couvrant un large éventail de problèmes de santé, voire de disciplines. Ces grandes cohortes que l'on qualifie de «généralistes » sont peu nombreuses en France. La cohorte «GAZ Électricité » (GAZEL) ${ }^{[5]}$ est la plus ancienne, elle a été mise en place en 1989 et est composée de 20500 agents d'Électricité de France-Gaz de France (EDF-GDF) âgés à l'origine de 35 à 50 ans qui sont suivis jusqu'à leur décès. Plus d'une trentaine de projets de recherche épidémiologique portant sur des thèmes très diversifiés ont été mis en place à partir de cette cohorte par une vingtaine d'équipes françaises et étrangères. De même, la cohorte française Étude longitudinale française depuis l'enfance (ELFE) ${ }^{[6]}$ a pour objectif de suivre 20000 enfants nés en 2011, de la naissance à l'âge adulte, en abordant de multiples aspects de la vie de l'enfant, sous l'angle des sciences sociales, de la santé et de la santé-environnement. Après la réalisation d'une étude pilote en 2007, le projet a été lancé en avril 2011, et est coordonné par l'unité mixte Institut national d'études démographiques (Ined) - Inserm - Établissement français du sang (EFS). Cette cohorte devrait permettre de comprendre ce qui perturbe ou au contraire favorise le développement de l'enfant, en analysant en particulier leur état de santé, leur alimentation, leur lieu d'habitation, mais aussi leur vie familiale et sociale.

Enfin, certaines cohortes sont conçues pour étudier les effets d'un traitement : exposition à un médicament ou un comportement de santé, comme pour la cohorte Nurses' Health Study ${ }^{[7]}$ mise en place aux États-Unis en 1976 dans le but initial d'étudier les effets à long terme de la contraception orale sur la santé. La cohorte Évaluation chez la femme enceinte des médicaments et de leur risque (EFEMERIS $)^{[8]}$ a pour but de détecter les médicaments à risque pour le fœtus et d'assurer un rôle d'alerte sur le plan des effets malformatifs mais également d'apporter des informations sur des médicaments encore mal évalués. Initiée en 2004, 48000 femmes, ayant accouché en Haute-Garonne, ont ainsi été incluses dans l'étude.

\section{Des efforts récents pour développer en France les outils épidémiologiques}

Une cohorte multithématique représente un investissement scientifique, technique, logistique et donc financier de longue durée. Idéalement, les études de cohortes nécessitent 3 types de financement :

- un financement de lancement nécessaire à la mise en place de l'infrastructure de recherche ;

- un financement stabilisé de maintien de la cohorte sur une durée d'au moins 3 à 5 ans ;

- des financements sur projets pour toutes les études qui viennent se greffer au fil du temps sur les données de la cohorte initiale.
Or, alors que la stabilité du financement est un élément essentiel à la réussite de tels projets scientifiques, beaucoup de projets de cohortes font l'objet de financements dont la pérennité n'est pas assurée et avec souvent peu de considération sur le potentiel intérêt collectif de ces projets.

Conscients de l'importance du développement des outils épidémiologiques dans la recherche en santé et des difficultés inhérentes au financement de ces projets de recherche de longue durée, plusieurs mesures ont été mises en œuvre ces dernières années.

Parmi celles-ci, le lancement des appels d'offres « cohortes nutrition-santé » ainsi que le dispositif des «très grandes infrastructures de recherche (TGIR) - Grandes cohortes en santé» initié en 2008 dans le cadre du « grand emprunt-investissements d'avenir ${ }^{[9]}$ ont permis le financement de plusieurs de ces cohortes nationales. De plus, dans le cadre du $4{ }^{\text {è }}$ Conseil stratégique des industries de santé en 2009, les acteurs publics et industriels ont réalise le « Portail épidémiologie France » qui propose un catalogue en ligne des principales bases de données en santé et études épidémiologiques menées en France. ${ }^{[10]}$

\section{Les enjeux respectifs des différents acteurs}

Une cohorte est un outil scientifique dont la mise en œuvre et l'utilisation des résultats ne peut concerner un acteur isolé. En effet, la bonne conduite d'un tel projet nécessite l'implication évidente des chercheurs, mais aussi souvent des industriels et des autorités de santé. Les enjeux respectifs de ces acteurs doivent être compris et pris en considération afin que les éléments d'information apportés puissent répondre aux questions de chacun.

Les chercheurs ont pour objectif de produire de la connaissance scientifique dans un domaine particulier. Ces derniers rapportent que le temps qu'ils consacrent à la gestion et à la logistique du projet empiète trop largement sur celui de l'investigation scientifique. De plus, il leur est difficile de s'assurer de la stabilité des moyens nécessaires à la réalisation du projet, dans la mesure où la valorisation scientifique est souvent décalée dans le temps de par la durée de ce type d'étude.

Les industriels ont pour objectif de mieux connaitre les données épidémiologiques françaises afin d'orienter les développements cliniques et de définir les populations qui pourraient bénéficier des traitements. Ils doivent aussi être en mesure d'estimer l'impact des médicaments mis à disposition sur les populations. Pour mener et financer ces études sur le territoire français, ils ont besoin d'infrastructures efficaces et veulent pouvoir bénéficier d'un accès large aux données disponibles. Ils regrettent souvent que les études qui leur sont demandées pour suivre les médicaments en situation réelle de prise en charge ne tiennent pas compte de l'existence des études et bases de données déjà mises en place. De plus, ils soulignent le fait que l'attractivité de la France pour les développements cliniques et les études de post-autorisation de 
mise sur le marché (AMM) dépend en partie de la manière dont sont organisées et gérées les cohortes en France. Le développement de l'épidémiologie est donc aussi un déterminant du potentiel économique national en santé.

Enfin les régulateurs doivent connaitre aux mieux la situation épidémiologique française afin de prendre les décisions adéquates. La mesure de l'état de santé de la population ainsi que l'évaluation des prises en charge sont nécessaires pour arbitrer entre les différentes stratégies de santé possibles. Les études épidémiologiques, et en particulier les cohortes, permettent de confirmer le bénéfice/ risque d'un médicament en situation réelle de traitement et d'évaluer l'efficience des nouvelles stratégies thérapeutiques.

\section{Propositions de la table ronde pour la mise en place de règles de bonnes pratiques visant à renforcer l'optimisation de la réalisation des cohortes épidémiologiques}

\section{1. Établir l'inventaire des études existantes: un préalable indispensable}

Le suivi de cohortes en épidémiologie nécessite des investissements de lancement et de maintien importants, ainsi que des compétences en épidémiologie qui sont encore peu présentes en France. C'est pourquoi, le choix d'initier une nouvelle cohorte en réponse à certaines questions de santé doit être apprécié en regard de toutes les données et études disponibles par ailleurs. Le portail «Épidémiologie France ${ }^{[10]}$ permet de réaliser un état des lieux de l'existant dans le domaine concerné, afin de juger si la constitution d'une nouvelle cohorte est nécessaire. Cependant, il se limite aux études françaises et l'accès aux données de celles-ci doit être discuté au cas par cas. De plus, parmi les questions de recherche formulées, toutes ne peuvent conduire à la mise en place de nouvelles études et a fortiori la constitution de cohortes; le choix doit être fait selon des priorités nationales de santé publique.

\subsection{La cohorte doit s'inscrire dans un projet collectif selon des règles bien établies}

Lorsque les données existantes ne permettent pas de répondre aux questions liées à des nouvelles problématiques de santé et qu'elle constitue une priorité en santé publique, alors la constitution d'une nouvelle cohorte peut être envisagée. Les étapes préliminaires sont essentielles car elles posent les bases d'une collaboration qui perdurera tout le long du suivi de la cohorte. Dans ce contexte, la création d'un consortium ou groupe de travail est une étape clé. En premier lieu, il faut identifier un investigateur principal adapté. Son rôle est d'initier, guider, motiver et organiser la mise en place du consortium. Il facilite la construction d'un consensus, aide à maintenir le calendrier, et rédige avec les membres un plan d'activités. Il devra laisser une liberté aux futurs membres pour définir leurs propres priorités et/ou intérêts, et adapter le consortium à leurs besoins. Il devra d'abord identifier les partenaires intéressés publics ou privés. Pour mener à bien cette étape, il est nécessaire de lister tous les acteurs (industriels, chercheurs et régulateurs) opérant dans le domaine cible du consortium. Après avoir informé les potentiels partenaires identifiés sur la nature du projet, l'étape suivante consistera à réaliser une enquête auprès d'eux afin de mieux connaitre leurs priorités et attentes. Il conviendra par la suite d'organiser des réunions entre les représentants des différentes structures qui restent intéressées par le projet, ceci dans le but de clarifier leurs objectifs et/ou intérêts. Ces réunions sont aussi le moyen de réfléchir sur les aspects légaux et sur les contributions respectives.

Un conseil scientifique constitué d'experts dans la pathologie ou le groupe de pathologies étudiées et de méthodologistes, réfléchit sur schéma d'étude, le nombre de patients à inclure et les détails du suivi. Une étude de faisabilité doit ensuite être menée à un stade précoce pour vérifier que l'étude est réalisable et en évaluer son budget.

Les réunions suivantes du consortium auront pour but de définir précisément la gouvernance du consortium. Les conditions d'accès aux données par les structures privées et publiques seront décidées collégialement lors de ces réunions et seront respectées tout au long de l'étude. Elles seront listées dans une charte d'accès aux données. Enfin, l'étude sera référencée sur le « portail épidémiologie France » afin que l'information diffuse le plus largement possible.

\section{Les demandes d'études de pharmaco- épidémiologie par les autorités de santé doivent faire l'objet d'une large démarche de concertation}

La pharmaco-épidémiologie est « une discipline mettant en application les méthodes et/ou le raisonnement épidémiologique pour évaluer, généralement sur de grandes populations, l'efficacité, le risque et l'usage des médicaments ».

Bien qu'il soit nécessaire de disposer d'études expérimentales spécifiques, la pharmaco-épidémiologie se veut observationnelle, ce qui s'inscrit dans une volonté de s'écarter le moins possible de la vie réelle afin de ne pas perturber les comportements habituels de prescription et de prises médicamenteuses.

Il est très fréquent que les autorités de santé demandent ce type d'étude aux industriels pour mesurer l'effet des médicaments en population. Il est même souvent stipulé qu'une nouvelle cohorte doit être conduite. Or, bien que les cohortes soient de très bons outils, il s'avère que l'information est parfois disponible dans des bases de données existantes ou que d'autres schémas d'études peuvent répondre aux questions posées.

Référencer ces données est indispensable. En effet, avant de mettre en place une étude de pharmaco-épidémiologie ad hoc, en 
général longue et coûteuse, il est important de vérifier que des données existantes ne permettent pas déjà de répondre à la question que l'on se pose.

Afin d'éviter que des études de pharmaco-épidémiologie soient menées alors que l'information existe au sein de cohortes existantes, il est nécessaire que les demandeurs (Direction générale de la santé [DGS], Haute autorité de santé [HAS], Groupe intérêt de santé publique et études post-inscritpion [ISPEP]), les industriels et les experts discutent et échangent avant la soumission d'une proposition de protocole. De plus, en cas de besoin d'une nouvelle étude, grâce aux échanges répétés, les méthodes d'évaluation les plus adaptées à la question posée peuvent être identifiées (nouvelle cohorte, étude ancillaire dans une cohorte existante ou utilisation d'une base de donnée). Au même titre que pour la constitution d'une cohorte non spécifique au médicament, les échanges préalables serviront aussi à évaluer rapidement les enjeux liés à la réalisation de l'étude choisie (faisabilité, financement, expertise disponible...) permettant ainsi d'associer tous les acteurs intéressés par la réponse à la question posée.

\section{Conclusion}

Les cohortes constituent un très bon outil épidémiologique qui permet de répondre à de nombreuses questions en santé. Néanmoins, c'est un outil coûteux, complexe et long à mettre en place. Bien que l'épidémiologie évolue en France dans un contexte institutionnel et de financement plus favorable depuis ces dernières années, la rareté des ressources et des expertises dans le domaine doit systématiquement conduire à s'interroger sur la pertinence des projets au regard des enjeux de l'ensemble des acteurs. Afin de maximiser l'efficience de ces projets de recherche, les participants de la table ronde rappellent qu'il est indispensable avant tout de mutualiser les connaissances afin que les cohortes puissent répondre en premier lieu aux enjeux de santé publique prioritaires. De plus, les participants ont jugé qu'il était nécessaire de développer de plus nombreuses interfaces entre les acteurs concernés (régulateurs, chercheurs et industriels) lorsqu'une question de santé publique ou l'introduction d'un nouveau médicament nécessite le développement d'un projet épidémiologique de cette envergure. Le renforcement de la communication et l'organisation des interactions entre les intéressés potentiels au projet de recherche est une condition nécessaire au développement de l'épidémiologie en France.

\section{Remerciements.}

Nous remercions chaleureusement les participants de la table ronde de Giens. Nous remercions sincèrement Martin Blachier pour sa participation à la rédaction du compte rendu de la table ronde.

\section{Participants.}

Yolande Adjibi (CIC UPCET, CHU, Marseille), Béatrice Augendre-Ferrante (Laboratoire Lilly), Marie-Noëlle Banzet (Laboratoire Servier), Laurent Becquemont (Inserm, Le KremlinBicêtre), Juliette Bloch (InVS), Brigitte Calles (Laboratoire Merck Sharpe \& Dohme Chibret), Geneviève Chene (Inserm, Université, Bordeaux), Françoise Clavel Chapelon (Inserm, Institut Gustave Roussy, Villejuif), Monique Couderc (Laboratoire Sanofi), Rima De Sahb (Laboratoire Roche), Pierre Ducimetiere (Inserm, Villejuif), Danièle Golinelli (Direction générale de la santé), Françoise Haramburu (CHU Bordeaux), Jean-Michel Joubert (UCB Pharma), Eléonore Lebrun (Inserm Transfert, Paris), Martine Pigeon (CNAMTS), Florence Scheck-Bine (Afssaps), Nathalie Schmidely (BristolMyersSquibb), Anne Solesse (Haute autorité de santé), Didier Veron (Ipsen), Dominique Vuillaume (Inserm, Paris), Guillaume Walckenaer (GlaxoSmithKline).

\section{Conflits d'intérêts. Aucun.}

Abréviations. AMM : autorisation de mise sur le marché ; DGS : Direction générale de la santé; E3N : Étude épidémiologique auprès des femmes de l'Éducation nationale ; EDF-GDF : électricité de France - gaz de France ; EFEMERIS : Évaluation chez la femme enceinte des médicaments et de leur risque ; EFS : Établissement français du sang; Elfe: Étude longitudinale depuis l'enfance ; EPIC : European prospective investigation into cancer ; GAZEL : (cohorte) Gaz Électricité : GMA : Groupe métholologies Alzheimer ; HAS : Haute autorité de santé ; Ined : Institut national d'études démographiques ; Inserm : Institut national de la recherche médicale; InVS : Institut national de veille sanitaire ; ISPEP : Groupe intérêt de santé publique et études post-inscription ; MEMENTO : deterMinants and Evolution of AlzheiMer's disEase aNd relaTed disOrders ; TGIR : très grandes infrastructures de recherche.

\section{Références}

1. Bouyer J. Méthodes statistiques - Médecine - Biologie. De Boeck Secundair; 2000. 364 p.

2. FondationPlanAlzheimer. Cohorte nationale de patients: MEMENTO [Internet]. http://www.fondation-alzheimer.org

3. Chambost H, Suzan F. Epidemiology of hereditary bleeding disorders: input of FranceCoag Network. Arch Pediatr 2010 juin; 17(6): 618-9

4. Clavel-Chapelon F, Jadand C, Goulard H, et al. E3N, a cohort study on cancer risk factors in MGEN women. Description of protocol, main characteristics and population. Bull Cancer 1996 déc; 83(12): 1008-13

5. Tran PD, Leclerc A, Chastang JF, et al. Prevalence of health problems in the Gazel (EDF-GDF) cohort: regional distribution and disparities. Rev Epidemiol Sante Publique 1994; 42(4): 285-300

6. Léridon H. A project of the French National Birth Cohort: ELFE. Arch Pediatr 2007 juin; 14(6): 702-4 
7. Colditz GA, Manson JE, Hankinson SE. The Nurses' Health Study: 20-year contribution to the understanding of health among women. J Womens Health 1997 Feb; 6(1): 49-62

8. Lacroix I, Hurault C, Sarramon MF, et al. Prescription of drugs during pregnancy: a study using EFEMERIS, the new French database. Eur J Clin Pharmacol 2009 août; 65(8): 839-46
9. Appel à projets Cohortes - France http://www.agence-nationale-recherche.fr

10. Portail Épidémiologie - France https://epidemiologie-france.aviesan.fr/

Correspondance et offprints: Jean-Marie Goehrs, JMGoehrs Partners, 85 avenue de Saint Cloud, 78000 Versailles, France.

E-mail : jeanmarie_goehrs@hotmail.fr 